\title{
2 Von den Grenzen des Lebendigen in der Naturphilosophie zu den Grenzen im Lachen und Weinen in der Kulturphilosophie
}

Nach der Vorstellung von Plessners Naturphilosophie im vorangegangenen Kapitel soll im nun kommenden Kapitel das Grenzthema exemplarisch vertieft werden. Es durchzieht die gesamte Philosophie Plessners. Wir hatten bereits in der Naturphilosophie gesehen, dass Plessner die Spezifik belebter gegenüber unbelebten Körpern aus dem Anschauungsbestand entwickelt, dass lebende Körper ihre eigene Grenze vollziehen. Er führt aber in seiner Naturphilosophie der Stufen des Organischen noch nicht den Anschauungsbestand in spezifisch humanen Phänomenen aus. Die exzentrische Positionalität wird dort noch aus einem Rückschlussverfahren erschlossen. Sie wird aus den biologischen und lebensweltlichen Leistungen, zwischen den verschiedenen non-humanen Lebensformen (Organisations- und Positionalitätsformen) unterscheiden zu können, als deren Ermöglichungsgrund rekonstruiert. Plessner explizierte den zu verschränkenden Bruch in der exzentrischen Positionalität erst später an den humanspezifischen Phänomenen des Lachens und Weinens als den Grenzen menschlicher Verhaltensbildung in dem gleichnamigen Buch von 1941, das damals in der Schweiz erschien, da er ab 1934 bereits in den Niederlanden im Exil leben musste.

Im Folgenden geht es aber nicht nur darum, den grenzthematischen $\mathrm{Zu}$ sammenhang seiner Naturphilosophie mit seiner Kulturphilosophie anhand der humanspezifischen Phänomene des Lachens und Weinens einzusehen. Plessner gehörte Mitte der 1950er Jahre auch $\mathrm{zu}$ den Initiatoren einer interdisziplinären Grenzforschung: Angesichts der Ausdifferenzierung des Wissenschaftsgefüges in immer mehr und vor allem methodisch verschiedenartigere Wissenschaftsdisziplinen schlug er als integrative Gegentendenz eine offene Grenzforschung vor. Die Frage nach der Universitas als der Einheit im Wissenschaftsgefüge müsse nicht mehr wie früher hierarchisch vorgegeben werden, etwa durch die Theologie im Mittelalter oder ein philosophisches System in der Neuzeit, wonach es dann die Universität in Fakultäten zu strukturieren galt, sondern könne als die Frage nach den Grenzen zwischen den verschiedenen Forschungsunternehmen neu gestellt werden, um nach Grenzübergängen zu suchen. Je nachdem, wovon diese Forschungsunternehmen in ihren Methoden, Modellen, Theorien gerade absehen, könne man durch Grenzfragen ihre ausge- 
blendeten Voraussetzungen, Bedingungen und Folgen thematisieren, um zu „grundsätzlichen Verklammerungen“" zwischen den Disziplinen zu gelangen. Keineswegs die einzige, aber eine interessante Möglichkeit, Grenzforschung anzulegen, bestehe darin, sie auf das anthropologische Problem zu fokussieren, inwiefern Menschen selbst zum Gegenstand der Forschung gemacht werden, ohne darin vollständig aufgehen zu können, da sie selbst auch diese Forschung leisten. Die Philosophische Anthropologie wurde hier als Rahmen für Grenzforschungen vorgeschlagen, in dem man von aktuellen Problemen wie z. B. der psychosomatischen Medizin ausgeht, um darum herum Natur-, Sozial- und Kulturwissenschaften einzubeziehen, sofern sie zu den offenen Grenzfragen des personalen Lebens von Menschen beitragen können. ${ }^{2}$

Plessner ist auf das Grenzthema nicht erst in den 1950er Jahren gekommen, um die Frage nach der Einheit der Universität durch ein neues Studium Generale für aufgeklärte Staatsbürger zu beantworten. Seine Philosophische Anthropologie war von Anfang an schon in den 1920er Jahren auf Grenzfragen nicht nur zwischen den verschiedenen Wissenschaftsdisziplinen und -gruppen ausgerichtet, sondern auch im Hinblick auf eine Lebenswelt, die alle Spezialisten miteinander in Zukunft teilen können und auf die hin seine Philosophie ihre Integrationsaufgabe versteht (siehe 5. Kapitel). Ich werde hier im Folgenden exemplarisch auf zwei Grenzverständnisse aus dieser Philosophischen Anthropologie eingehen. Zum einen spielt der Grenzbegriff eine große Rolle in der Naturphilosophie, wenn man sich fragt, wie sich belebte Körper im Unterschied zu unbelebten Körpern verhalten. Zum anderen kommt das Thema bereits im Untertitel von Plessners Buch über das Lachen und Weinen als den interkulturell geteilten Grenzen menschlichen Verhaltens vor. Bevor ich mich diesen beiden Grenzphänomenen in der Naturphilosophie (2.2.) und Kulturphilosophie (2.3.) zuwende, sollte ich aber noch kurz einführend erläutern, was hier überhaupt unter Philosophischer Anthropologie, ihrem Denkeinsatz und ihrem methodischen Zugang zum Leben, verstanden wird (2.1.).

1 Helmuth Plessner (1983m): Über einige Motive der Philosophischen Anthropologie, 120-123, Zitat: 120. Dieser Aufsatz erschien ursprünglich im Studium Generale 1956.

2 Siehe ebd., 124-125. In diesem Sinne habe ich Grenzfragen zwischen der neurobiologischen Hirnforschung und der vergleichenden Verhaltensforschung gestellt in Krüger (2010). 


\subsection{Der methodische Zugang zum Leben in der Philosophischen Anthropologie durch Neutralisierung des Verfahrens gegen dualistische Vorurteile}

Es gibt viele erfahrungswissenschaftlich ausgerichtete Anthropologien wie die biologische Anthropologie, die medizinische Anthropologie, die Sozial- und Kultur-Anthropologie, die historische Anthropologie, die sich alle aus ihrer jeweils spezifischen Sicht damit beschäftigen, was für Lebewesen der Gattung Mensch wesentlich und spezifisch ist. „Philosophisch“ bedeutet dann die Hervorhebung einer integrativen Sicht: Wie hängen das Naturwesen Mensch mit dem Sozial- und Kulturwesen Mensch historisch und systematisch gesehen zusammen? Die philosophische Anthropologie hat sich seit Johann Gottfried Herder, ihrem Begründer in der 2. Hälfte des 18. Jahrhunderts, für die integrative Ganzheitlichkeit des Menschen gegen seine disziplinären Aufteilungen, gegen seine ethnozentrischen Vorurteile gegenüber anderen Kulturen und gegen seine speziesistischen Vorurteile gegenüber anderen Arten von Lebewesen interessiert. Es geht ihr nicht um exklusive Selbstüberschätzungen von Menschen gegenüber anderen Menschen und Lebewesen, sondern um den integrativen Zusammenhang der eigenen Homininen-Art mit anderen Spezies und anderen Kulturen und Gesellschaften in der Zukunft. Andererseits handelt es sich aber in der Philosophischen Anthropologie auch nicht einfach um eine Gleichmacherei aller Arten und Weisen zu leben und darunter als Mensch zu leben. Die Nivellierung aller Unterschiede scheint einer moralischen Gleichstellung verschiedener Lebensformen zu dienen, ist aber praktisch betrachtet bequem und lenkt davon ab, diejenige Verantwortung für andere Lebewesen und die ökologisch gefährliche Lage auf der Erde zu übernehmen, die nur personale Lebewesen in ihren Lebensformen im Unterschied zu anderen Primaten oder Säugern übernehmen können.

Philosophische Anthropologie hieß und heißt auch Kritik an Anthropomorphismen, d. h. Kritik an der Übertragung von einem spezifisch menschlichen Selbstverständnis auf das Verständnis anderen Lebens und der Welt überhaupt. Ein Extremfall des Anthropomorphismus ist der Narzissmus in der westlichen Kultur, wie er seit Christopher Laschs gleichnamigem Buch immer wieder beschrieben worden ist (Lasch 1995). Narzisse schon im umgangssprachlichen Sinne können sich überhaupt nicht vorstellen, dass Leben und Welt etwas anderes sein könnten als Spiegel für das von ihnen heiß geliebte Selbstbild des Eigenen. Um solche Zirkel im Selbstverstehen vermeiden zu können, setzt die Philosophische Anthropologie ihre Untersuchungen gerade nicht direkt beim Menschen an, sondern auf Umwegen bei der nicht spezifisch humanen Natur, 
der unbelebten und belebten Natur. Man beginnt also philosophisch gesehen gerade beim Anderen und Fremden, nicht mit dem Eigenen, um aus dem Zirkel des Eigenen heraus $\mathrm{zu}$ gelangen und letztlich den Zusammenhang zwischen Eigenem, Anderem und Fremden herausfinden zu können.

In der westlichen Kultur seit der Neuzeit hat sich, im Vergleich mit anderen Kulturen der Menschheit, philosophisch betrachtet die Vorherrschaft des Selbstbewusstseins wirkungsgeschichtlich gesehen durchgesetzt, unabhängig von den besten Intentionen einzelner Autoren: Ich denke, also bin ich, wie Descartes schrieb; das Ich, das alle meine Vorstellungen und Gedanken begleiten können muss, wie Kant es ausdrückte. Diese Synthese im Selbstbewusstsein schien eine unbezweifelbare Begründung rationaler Erkenntnis zu ermöglichen. Dieses Ich spielte in seiner Wirkungsgeschichte seit nunmehr auch über 200 Jahren nicht nur epistemologisch, also für die Wissenserzeugung, eine privilegierte Rolle. Es scheint auch ontologisch, d. h. unter allen möglichen Weisen zu sein, sich selbst dadurch auszuzeichnen, dass es andere Weisen zu leben und zu sein von sich ausschließt und zu beherrschen sucht, indem es durch Machtgefüge die ihm entsprechenden Rollenmuster der ersten Person Singular gegenüber allen anderen pronominal möglichen Rollen im Singular und Plural privilegiert. Die Philosophische Anthropologie war daher von Beginn an mit einer Kritik an diesem dualistisch abgespaltenen Subjekt und seiner Selbstprivilegierung im Ich in Erkenntnistheorie und Ontologie beschäftigt.

Der ontologische Dualismus besagt kurz Folgendes: Etwas müsse entweder wie die res extensa, also ausgedehnt im physikalischen Raum und der physikalischen Zeit sein, oder wie die res cogitans sein, also etwas Denkendes, das um sich weiß. Laut dieser exklusiven Alternative Entweder - Oder dürfte es Leben überhaupt nicht geben. Was dem Dualismus entsprechend gegeben sein darf, müsste entweder mechanisch manipulierbar und berechenbar sein, oder es müsste sich selbst evident, sich selbst Zweck und nach reflexiver Regel verfahrend sein. Dazwischen, darüber, darunter, außerhalb dieser Alternative dürfte es nichts geben. Der Zusammenhang der beiden Seiten, die sich ausschliessen, grenzt an ein Wunder, das wir vorgängig oder bei Gelegenheit bei René Descartes noch unserem christlichen Gott verdanken müssen. Diese dualistische Exklusion, die sich der eigenen religiösen Mission in Descartes noch sicher blieb, war nach Darwin, inmitten der Säkularisierung und Modernisierung nach dem ersten Weltkrieg in den 1920er Jahren, für viele nicht mehr resonanzfähig. Menschen schienen aus dem Leben zu kommen und vor die Aufgabe gestellt, sich in demselben zu halten, besser oder schlechter. Ihre Selbstermächtigungen, aber auch ihre Selbsterfüllungen nahmen im Diesseits zu, die Glaubenssi- 
cherheit ans Jenseits ab, von den Spaltungen innerhalb der religiösen Glaubensformen noch abgesehen.

Auf diese Herausforderung antwortete Max Scheler, der historisch die Philosophische Anthropologie neu begründete, indem er die Exklusivität der dualistischen Alternative negierte. Diese Negation nannte er die Neutralisierung des methodischen Verfahrens gegen den exklusiven Dualismus: Aus entweder materiell oder geistig wird: Was sowohl materiell als auch geistig ist, kann leben. Aus entweder physisch oder psychisch wird: Was sowohl physisch als auch psychisch ist, kandidiert fürs Leben (siehe Scheler 1985, 10, 162; Scheler 1986, 18f., 39-42). Woran erkennt man aber die Einheit von Physischem und Psychischem, die Einheit von Materiellem und Geistigem?: Man erkennt diese Einheiten am lebendigen Verhalten, wenn man es nicht auf Reflexe reduziert, sondern es auch nach der Sinngemäßheit seiner Richtungen als Phänomen beobachtet (Buytendijk/Plessner 1982c). Plessner schloss methodisch direkt an Schelers Neutralisierung des Verfahrens gegen die üblichen dualistischen Vorurteile an und leistete in seiner Freilegung des Verhaltens mit Scheler einen Doppelschlag, einerseits gegen die Behavioristen, andererseits gegen den dualistischen Rückfall von Husserl selbst in das transzendentale Selbstbewusstsein (in dessen Ideen zu einer reinen Phänomenologie und phänomenologischen Philosophie, 1913). Der Verhaltensbegriff wird zentral, weil er Leben über den Organismus hinaus als gerichtete Beziehung zur Umwelt fasst, von der her Rückbeziehungen möglich sind. Leben ist so verstanden immer auch organisch, aber nicht nur organisch, sondern so organisch, dass sich der Organismus in seiner Umwelt verhalten kann, also in der Generationenfolge und als Individuum einer Art an einem übergreifenden Lebensprozess teilzunehmen vermag. Im Kern besteht hier Leben aus übergestalthaften ${ }^{3}$ Ganzheiten von Teilen, also Organismen, und den Verhaltensweisen solcher Organismen in ihren Umwelten, die sich zur Ganzheitlichkeit des Lebensprozesses erweitern lassen. Im Gegensatz zu Scheler, der die Philosophische Anthropologie noch im Rahmen einer spekulativen Seinsmetaphysik begründet hatte, fundierte Plessner in seinem Buch Die Stufen des Organischen (1928, hier 1975) die Philosophische Anthropologie

3 Laut der Gestalttheorie von Wolfgang Köhler gibt es übersummenhafte Ganzheiten bereits in der unbelebten Natur. Man würde heute von einer Selbstorganisation im physikalischchemischen Sinne sprechen, von der es die biologische Selbstproduktion und -reproduktion zu unterscheiden gilt. Siehe Krüger (1993, erster Teil). Da Plessner Köhlers Gestalttheorie unterstellte, sprach er von „übergestalthafter“, d. h. organismischer Ganzheit, um an die Spezifik lebender Körper heranzukommen (Plessner 1975, 96-99). 
durch eine Naturphilosophie. ${ }^{4}$ Damit komme ich nun zu diesem, wie angekündigt, ersten Grenzverständnis.

\subsection{Naturphilosophisches Grenzverständnis: Lebendige Körper unterscheiden sich von unbelebten Körpern dadurch, dass sie auch ihre eigene Grenze realisieren}

Stellen Sie sich zum Anfang bitte die Aufgabe vor, belebte Körper von unbelebten Körpern unterscheiden zu müssen. Was geschieht in dieser Unterscheidung auf Seiten des Gegenstandes? Nehmen wir dafür zwei Beispiele, eines für einen unbelebten Körper, der also nicht wie ein Organismus lebensfähig ist, und eines für einen belebten Körper, d. h. einen Organismus. Stellen Sie sich zunächst einen Handschuh vor, den Sie überziehen. Er wärmt Ihre Hände. Und nun versuchen Sie mal das Innere des Handschuhs nach außen zu kehren. Sie ziehen das innere Futter mit Ihren Fingernägeln und Fingerkuppen vorsichtig nach außen, so dass die Außenoberfläche des Handschuhs nach innen wandert, gleichsam zu dem neuen Futter wird. Als ob Sie z. B. den Handschuh waschen wollten. Und jetzt wiederholen Sie das Ganze. Was jetzt innen ist, ziehen Sie wieder nach außen, so dass das, was außen ist, wieder nach innen gedreht wird. Am Ende haben Sie den alten Ausgangszustand wiederhergestellt. Und nun versuchen Sie die ganze Prozedur mal mit einem Kaninchen. - Wie bitte? Das geht nicht, sagen Sie. Ja, das könnte etwas mit dem Unterschied zwischen belebten und unbelebten Körpern, die keine Organismen sind, zu tun haben. Lebende Körper lassen sich nicht reversibel umstülpen. Sie haben etwas Irreversibles, für sie Unbedingtes, Absolutes, das, hat man es erst einmal zerstört, nicht einfach mehr umgekehrt werden kann. Wenn man ihnen nicht selbst das Regime zwischen ihrem Innen und Außen überlässt, wenn man sie wie etwas Mechanisches von außen umstülpt, ohne dass sie es selbst reparieren können, dann sterben sie. Sie realisieren ihre eigene Grenze, darin besteht die hypothetische Intuition. Diese Realisierung kann man ihnen nicht abnehmen, ohne sie zu töten.

Plessner erprobt diese Intuition zunächst in der lebensweltlichen Anschauung, wie wir alle sie miteinander teilen, ohne dafür eine spezielle Ausbildung mit besonderen Labortechniken zur Verfügung zu haben. Wenn man sich fragt, wie die Anschauung von bestimmten unbelebten, $\mathrm{d}$. h. nicht gerade toten, son-

4 Zum Zusammenhang und Gegensatz zwischen Schelers und Plessners Philosophischer Anthropologien siehe Krüger (2009a, 6. Kap.). 
dern prinzipiell nicht lebensfähigen Körpern in Raum und Zeit gelingen kann, dann scheint das angeschaute Ding aus einem begrifflich artikulierten Kern zu bestehen, der einen Mantel von anschaulichen Eigenschaften trägt. Der Kern wird als Satzsubjekt ausgedrückt, dem man Eigenschaften prädiziert. Was man hier und jetzt wahrnimmt, sind diese empirischen Eigenschaften, die man im Gegensatz zu anderen Eigenschaften bestimmen kann. Sie können im Nacheinander der Zeit und im Nebeneinander des Raumes wechseln, ohne dass sich der Dingkern als die Einheit des Dinges auflösen müsste. Täte er dies, handelte es sich um eine andere Dingart. Irgendwie müssen die Dingkerne und ihre Eigenschaftsmäntel nach Arten miteinander zusammenhängen. Der Kern scheint vom Inneren seiner Eigenschaften her, die wir von außen beobachten können, ihre bestimmte Einheit zu tragen. Sie kann sich in Gestaltsprüngen (stehendes Wasser gefriert) und in Artsprüngen komplizieren, ohne dass man für den Wechsel zwischen Kern und Eigenschaften andere als im lebensweltlichen Sinne mechanisch handhabbare Kräfte erwarten würde.

Fragt man sich weiter, wie die Anschauung von bestimmten belebten Körpern in Raum und Zeit gelingen kann, geschieht in einer solchen Anschauung mehr als ein Gestalten- und Artensprung innerhalb des Unbelebten. Lässt man dem belebten Körper Raum und Zeit, damit er sich in seinen Bewegungen oder sonstigen Verhaltensweisen zeigen kann, entfaltet sich in dem Wechsel zwischen seinem Kern und seinen Eigenschaften eine innere Dynamik, die sich auch in seinem Äußeren auf eine ganzheitliche, nicht einfach die Summe von Atomen ausmachende Weise entfaltet. Sie greift sowohl nach innen als auch nach außen Platz und ufert aus in ihrer Zeit. Die ganzheitliche Dynamik des Lebendigen behauptet sich, wie man heute meistens sagt, als Eigenraum und Eigenzeit, ohne dass sie aufhört, an die Bedingtheiten der unbelebten Körper gebunden zu bleiben, so im Stoffwechsel und Energieaustausch, d. h. in ihrer Abhängigkeit von Medien ihrer Umgebung. Wie lässt sich dieser - lebensweltlich nicht ersetzbare - Sprung in der Anschauung von belebten gegenüber unbelebten Dingen einsichtig machen, in dem wir so etwas wie einen dem Belebten selbst zukommenden Spielraum und eine ihm zukommende Spielzeit vergegenwärtigen?

Für die Beantwortung dieser Frage entwickelt Plessner seine Hypothese, die Spezifik der Lebendigkeit von Körpern bestehe darin, dass ihnen nicht nur wie den unbelebten Körpern Konturen als eine von außen beobachtbare Grenze zukommen, sondern dass sie ihre eigene Grenze realisieren. Gehen wir, um diese Hypothese zu verstehen, noch einmal auf die unbelebten Körper zurück. Diejenigen, die bereits das Kapitel 1.4. gelesen haben, bitte ich um Verständnis dafür, dass sich nun einiges hier unter 2.2. wiederholen wird. 
Die Bewegungsrichtungen zwischen unbelebten Körpern in Raum und Zeit finden in bestimmten Konturen Anhaltspunkte für die Unterscheidung der Körper nach Dingarten. Relativ auf einen bestimmten unbelebten Körper bezogen lassen sich die divergenten Richtungen nach außen und nach innen anhand seiner Konturen differenzieren. Diese Unterscheidung ist aber relational umkehrbar auch für alle anderen unbelebten Körper zu treffen. Die Bewegungsrichtungen und ihre Relationen gehen durch alle Körper hindurch. Unbelebte Körper hören da auf, wo andere Körper oder Medien ihrer Umgebung beginnen, und sie beginnen da, wo andere Körper oder Medien ihrer Umgebung aufhören. Die Grenze erscheint als der Übergang zwischen einem Körper und dem Medium, an das er stößt. Das „Zwischen“ zwischen Körper und Medium ist „leer“ im Sinne des leeren Raumes und der leeren Zeit. Der Grenzübergang gehört weder dem Körper noch dem Medium an, sofern es sich aus den Koordinaten einer unterstellten Raumzeit als dem Rahmen ergibt. Dieses Dazwischen gehört aber im Sinne mechanischer Gesetze beiden, Körper und Medium, an, insoweit diese Regelmäßigkeiten sowohl in den Körpern als auch in den Medien ihrer Umgebung wirken. Unbelebte Körper bilden Konturen aus, die in der Lebenswelt mechanisch handhabbaren Gesetzen unterliegen, aber diese Konturen dienen nicht der Funktion, den jeweiligen Körper im Ganzen, d. h. übergestalthaft und übersummenhaft, zu begrenzen (siehe Plessner 1975, 103f.).

In dieser Frage nach der Funktion der Konturen für das Ganze besteht aber die Frage nach dem organismischen Charakter des Körpers, d. h. ob er einen Organismus bildet. Stehen Konturen in diesem Funktionszusammenhang, dann sind sie nicht nur im Sinne lebensweltlich geläufiger mechanischer Bewegungsrichtungen Anhaltspunkte dafür, wo und wann der unbelebte Körper anfängt und aufhört. Gehört der Rand zu dieser Grenzfunktion für eine Integration in ein Ganzes, gibt es ein Außen und Innen des betreffenden Körpers so, dass Außen und Innen ihm in seinem Vollzug zugehören. Er staut und lockert sich nach innen und verhält sich nach außen, z. B. als reizbar, angezogen oder zurückweichend. Im Falle der Realisierung der eigenen Grenze schließt sich der Körper gegen das ihm Äußere, das „ihm entgegen ist“ (ebd., 127f.), ab und auf. Gleichursprünglich verschließt und öffnet er damit sein Inneres, dem er auch von außen zurückkommend entgegen ist. Dadurch dass der lebende Körper seine eigene Grenze im Verhalten vollzieht, sich für außen öffnet und abschließt, sich nach innen abschließt und öffnet, gewinnt er einen Eigenraum und eine Eigenzeit, ein eigenes Spiel von Verhaltensmöglichkeiten, im Unterschied zu seiner Umgebung. Erinnern Sie sich zum Beispiel an Ihre Beobachtung von Bakterien unter dem Elektronenmikroskop in Ihrer Schulzeit, wie diese Winzlinge zwi- 
schen Ruhelage und pulsierenden Bewegungen der Flucht vor und der Anziehung durch etwas wechseln.

Ein Körper, der seine eigene Grenze verwirklicht, vollzieht selbst den Grenzübergang zwischen seinem Inneren und Äußeren in beide Richtungen, dem Sinne nach sowohl von innen nach außen als auch von außen nach innen. Aber auch wenn man sich solche Anschauungen leicht vorstellen kann, so weiß man doch, dass Anschauungen auch täuschen können. Daher fragt Plessner weiter, welche Ontologie wir annehmen müssen für den Fall, dass die Anschauung Wirklichkeit und nicht bloß ein Schein ist, wie im Falle einer sich plötzlich öffnenden Schlange aus Papier. Wenn die Anschauung davon, dass der Vollzug des Grenzüberganges dem lebenden Körper selbst zukommt, wirklich ist, dann geht er tatsächlich über sich hinaus ins ihm Äußere und kommt von dort zu sich zurück, kurz: er positioniert sich in seiner Umgebung. Und er geht gleichursprünglich in sich hinein ins Innere und kommt von dort zurück ins Äußere, kurz: er organisiert sich. In seinem „positionalen Charakter oder seiner Positionalität“ (ebd., 129) bestimmen

die Momente des ,über ihm Hinaus‘ und das ,ihm Entgegen, in ihn Hinein' ein spezifisches Sein des belebten Körpers, das im Grenzdurchgang angehoben und dadurch setzbar wird. In den spezifischen Weisen ,über ihm hinaus‘ und ,ihm entgegen“ wird der Körper von ihm abgehoben und zu ihm in Beziehung gebracht, strenger gesagt: ist der Körper außerhalb und innerhalb seiner. Der unbelebte Körper ist von dieser Komplikation frei. (Ebd.)

Der belebte Körper ist nicht nur ein „bloßes Ding, sondern ein Wesen“, das auch für sich ist, eben ein „Lebewesen“ (ebd., 131). Es verhält sich zu der Stelle, an der er sich im leeren Raum befindet, „raumhaft“, indem er seinen Raum auf ihm unumkehrbare Weise erfüllt und behauptet (ebd., 312). Das Lebewesen verhält sich zu der Gegenwart, in der es sich als Körper in der leeren Zeit befindet, „zeithaft“, indem es diese Gegenwart auf ihm irreversible Weise erfüllt und behauptet (ebd., 175-177).

Ein Körper, der sich (dem Sinne seiner Verhaltensrichtungen nach von innen und nach innen) organisiert und (von außen und nach außen) positioniert, hört nicht auf, auch ein unbelebter Körper zu sein. Insofern er im spezifizierenden Sinne lebt, muss er dasselbe Wesen bleiben können in allen möglichen Grenzübergängen. Insofern er auch aus unbelebten Körpern besteht, muss er das gleiche bleiben, d. h. eine Kombination von Körpern, die für das lebendige Ganze funktional äquivalent ist. Insoweit er dasselbe und das gleiche bleibt, führt er Tautologien auf. Diese müssten aber, im Sinne einer lebensnötigen Möglichkeit in anderer Hinsicht mit Paradoxa zusammenfallen. Dasselbe ist nicht das Gleiche und umgekehrt. Das Äußere ist nicht das Innere und umge- 
kehrt. Das Lebendige ist nicht das Unbelebte und umgekehrt. Die Bewegungsrichtungen bleiben divergent und reversibel, auch wenn sie im Vollzug des Lebewesens dem Sinne nach konvergieren und unumkehrbar werden: „Bleiben, was es ist, Übergehen in das, was es nicht ist (über ihm hinaus) und in das, was es ist (in ihm hinein)“ (Plessner 1975, 138).

Wie könnte es möglich sein, dass sich diese Tautologien und jene Paradoxa, die doch einen Widerspruch bilden, im Raum und in der Zeit raumhaft und zeithaft ereignen können? - Indem sie sich im Raum und in der Zeit auf verschiedene Stellen verteilen, die raumhaft, d. h. als Eigenraum, und zeithaft, d. h. als Eigenzeit, durch Organisation und Positionierung integriert werden. Unterhalb dieser Komplexion zum Ganzen im Prozess ist es unmöglich, Leben in einer Ontologie des Wirklichen verständlich werden zu lassen. Leben ist nicht einfach ein Seiendes, sondern ein Werdendes: „Ein Ding positionalen Charakters kann nur sein, indem es wird; der Prozess ist die Weise seines Seins“ (ebd., 132).

Insofern in einem solchen Prozess das Ausgangsetwas zu einem Endetwas wird, ist er gerichtet: „Die Synthese findet als eine besonders gerichtete Form des modus procedendi statt: als Entwicklung“ (ebd., 140). Um diese Entwicklungsrichtung des Prozesses zu verstehen, überzeugt die Annahme nicht, es gehe nur um den Modus eines bloßen „Vermögens und Könnens“, die Kannqualität des „Könnens oder Nicht-Könnens“: Dann denke man, so Plessner, noch dualistisch, zu der Seinsweise des Körpers komme aus unserem Selbstverständnis ein Können oder Vermögen irgendwie hinzu. „Kannqualität als Seinsqualität, seiende Möglichkeit gilt es zu begreifen“ (ebd., 172). Diese seiende Möglichkeit besteht nicht in der Art und Weise von Möglichkeit, die man mit dem Lebewesen machen kann (,Mit-ihm-Möglichkeit“), sondern in einer Art und Weise von Möglichkeit, die an dem lebenden Körper selbst vorkommt, in einer „An-ihm-Möglichkeit des physischen Dinges“ (ebd., 174). Wolle man dieses Möglichsein unabhängig von unserer eigenen Teleologie, wie man durch Handlungen etwas determiniert, verstehen, bezeichne es nur eine „besondere Richtung vom Nichtsein zum Sein, die in dem Wort Noch Nicht festgehalten wird“ (ebd., 176) und die Einheit des Körpers in der Zukunft betrifft:

Möglichkeit fasst also eine Richtungseinheit, die gegen die Bestimmtheitsrichtung des Seienden in der Zeit Vergangenheit Gegenwart Zukunft gekehrt ist. Im Können des Seins wird letztlich nichts anderes als ein Vorwegverhältnis statuiert, in welchem die Abhängigkeitsrichtung von der Zukunft zur Gegenwart läuft. (Ebd.)

Dafür muss man annehmen, soll es mit der üblichen zeitlichen Determinationsrichtung verträglich bleiben, dass zwischen den Erfüllungen der Zeitmodi ein „zeithaftes“ Verhältnis stattfinde, das nicht innerhalb der leeren Zeit liege, also 
von ihrem Standpunkt aus als „zeitlos“ erscheint: „Erst dem lebendigen Sein ist Zeit in ihren Modis wesenhaft, denn es konstituiert sich vermittels der Zeit, insofern es ein Sein bedeutet, das ihm selbst vorweg ist“ (Plessner 1975, 179). Es werde nicht ein Bestimmtes vorweggenommen, das erst noch kommen werde, sondern ein Selbstbezug, der als Erfüllung der Zukunft in der konkreten Gegenwart bestimmbar werde. „Das ,Ihm selbst Vorweg' und das lebendige Sein besagen ein und dasselbe. Also ist lebendiges Sein ebenso sehr ihm selbst nach oder Erfüllung seiner selbst“ (ebd., 180). Es lebt im Modus seiner Fundierung aus der Zukunft in seiner konkreten Gegenwart, d. h. im Modus seiner „erfüllten Potentialität“ (ebd.).

Für den hiesigen Zweck muss dieser kurze Exkurs in Plessners naturphilosophisches Grenzverständnis genügen. Die Intuition, dass lebende Körper im Unterschied zu unbelebten Körpern ihre eigene Grenze vollziehen, sollte nur für die Anschauung plausibel gemacht und zu der Frage entfaltet werden, in welcher Ontologie diese Anschauung als Wirklichkeit verständlich werden könnte. Dabei führt Plessner dasjenige, was ich einleitend als den Eigenraum und die Eigenzeit des Lebendigen bezeichnet hatte, als das Raum- und Zeithafte im Unterschied zum leeren Raum und leerer Zeit aus. Ob sich die philosophische Hypothese auch erfahrungswissenschaftlich bewährt, ist vor allem die Frage danach, ob sich die Fakten (Ontik) und deren Interpretation (fachspezifische Ontologie) in den relevanten Erfahrungswissenschaften, hier also vor allem der Biologie und Medizin, im Rahmen der vorgeschlagenen naturphilosophischen Ontologie verstehen lassen. Diese Ontologie führt Plessner als eine monographische „Deduktion“ von nicht nur indikatorischen, also Lebendiges in der Anschauung anzeigenden, sondern konstitutiven, also wirklichen Wesensmerkmalen für Lebenssphären aus. Sie führt von offenen und geschlossenen Organisationsformen über zentrische Positionalitätsformen bis zu exzentrischen Positionalitätsformen, die personales Leben in einer Welt im Unterschied zu Umwelten ermöglichen (siehe 1. Kapitel).

\subsection{Kulturphilosophisches Grenzverständnis: Personale Lebewesen erfahren ihre Verhaltensgrenzen im ungespielten Lachen und Weinen}

Auch hier besteht die Aufgabe erneut darin, dass man sich die relevanten Phänomene, d. h. die qualitativ gemachten Erfahrungen nun des Lachens und des Weinens, immer wieder vorstellen muss, um die eigene Anschauung zu aktivieren, sie beschreiben und deuten zu können. So gelangt man wie schon in der 
Naturphilosophie jetzt auch in der Kulturphilosophie in den methodischen Viererschritt der phänomenologischen Beschreibung, der hermeneutischen Deutung und der dialektischen Entfaltung des Phänomens zu seinem Widerspruch, bis man am Ende danach fragen kann, was man insgesamt an Ermöglichungsstrukturen für die Begegnung mit dem Phänomen in Anspruch genommen hat. Das Interessante an den Phänomenen des Lachens und des Weinens liegt darin, dass sie in allen Kulturen von Menschen vorkommen. Von Kindesbeinen an wird das spezifisch menschliche Verhaltensrepertoire zwischen ihnen erlernt. Wie kulturgeschichtliche und humanethologische, sozial- und kulturanthropologische Studien zeigen, kann man diese Phänomene auch in einem selber fremden Kulturen als solche erkennen, wenngleich wie bei allen Erkenntnissen auf eine fehlbare Weise, und auch in einem oberflächlichen Sinne deuten, insoweit einem die Situation als Kontext dieser Phänomene zugänglich wird. Sie werden zwar in jeder Kultur anders stilisiert und von jedem individuell variiert, setzen aber doch am Verhältnis zwischen dem leiblichen Ausdruck und der Körperbeherrschung von Personen an, wodurch sie allgemein zugänglich werden. ${ }^{5}$

In dem Koordinatensystem von Plessners Philosophischer Anthropologie existieren personale Lebewesen in zwei Reihen von Relationen: Einerseits durch Rollen, also durch soziokulturelle Medien, in einem Verhältnis zu anderen Personen, in dem sie sich miteinander und gegeneinander tragen, wodurch sie eine Mitwelt bilden. Andererseits existiert auch jede Person in ihrer Körper-LeibDifferenz in einem doppeldeutigen Verhältnis zu ihrem eigenen Organismus. Insoweit die Person ihren Organismus für bestimmte Aufgaben instrumentieren und als Medium verwenden kann, spricht Plessner davon, dass die Person ihren Körper wie andere Körper auch zu haben vermag. Insofern wird der Person auch ihr eigener Körper vertretbar, austauschbar oder sogar ersetzbar, etwa in Prothesen. Insoweit aber die Person in ihrer Lebensführung ihren Organismus nicht vollständig vertreten, austauschen oder gar ersetzen kann, lebt sie in ihm und mit ihm als Leib. Indem sie ihn vollzieht, ist sie Leib. Weder das KörperHaben noch das Leib-Sein ist vollständig für die jeweils lebende Person: Der Mensch, der soziokulturell als Person angesprochen wird, ,ist weder allein Leib noch hat er allein Leib (Körper). Jede Beanspruchung seiner physischen Existenz verlangt einen Ausgleich zwischen Sein und Haben, Draußen und Drinnen“ (Plessner 1982f, 241). Der Leib stellt - im Ergebnis seiner zentrischen Or-

5 Siehe zur Kulturgeschichte des Lachens im Abendland seit der griechischen Antike Prütting (2013). Vgl. zur Ritualisierung des Weinens im Kontext des Todes Ariès (1982). Vgl. aus der Sicht der vergleichenden Verhaltensforschung Eibl-Eibesfeldt (1997). 
ganisation und seiner Habitualisierungsgeschichte - von sich her auf konzentrische Weise Verhaltenseinheiten mit seiner Umwelt her, die über den Organismus in dessen Umwelt hinausgehen. Die Person aber steht exzentrisch aus dieser Konzentrik des Leibes heraus in der Mitwelt, d. h. in den Relationen zu anderen Personen. Insofern sie sich exzentriert, kann sie ihren Organismus als Körper haben. Insoweit sie sich rezentriert, also in ihrem Leibsein aufgeht, vermag sie es nicht, ihren Organismus als Körper zu handhaben. In personalen Lebensformen ist somit erwartbar, dass eine Person je nach ihrer Rolle im mitweltlichen Verhältnis zu anderen Personen einen Ausgleich zwischen ihrem Leibsein und Körperhaben aufführen kann. ${ }^{6}$ Die Phänomene des Lachens und Weinens fallen nun in der Anschauung gerade dadurch und in dem Maße auf, als in ihnen eine Person diesen erwartbaren Ausgleich in Situationen nicht zustande bringt. Leibsein und Körperhaben laufen ihr hier und jetzt auseinander, obgleich sie ansonsten beide Verhaltensweisen gegenüber ihrem Organismus nach Rollenmustern zu integrieren vermag.

Dieses Auseinanderfallen von Leibsein und Körperhaben verlangt eine Deutung, die hier nur angerissen, nicht ausgeführt werden kann, weil dies letztlich eine dichte Analyse der betreffenden Kultur und individuellen Lebensgeschichte und einen interkulturellen Vergleich erfordern würde. Bleiben wir exemplarisch aus Zeitgründen in unserer eigenen Kulturtradition als einer eigenen Hermeneutik stehen: Wenn Menschen auf ihre Verhaltensprobleme antworten können, dann tun sie dies zumeist, indem sie in der entsprechenden Verhaltenssituation mehr oder minder angemessen handeln, sprechen und variabel gestalten können. Gibt es Streit, versucht man gute Gründe für die Handlungs-, Sprach- und Gestaltungsweise als situativ angemessene $\mathrm{zu}$ artikulieren. Die Situationen sind vorstrukturiert als alltägliche oder außeralltägliche, familiäre, berufliche, private, öffentliche, ökonomische oder politische, wofür es die Erwartungen gibt, dass bestimmte Rollenmuster ausgeübt werden. Man spielt eine Rolle als Eltern, Kinder, Vorgesetzte und Untergeordnete, Steuer- und Staatsbürger, Unternehmer oder Konsument, Provider oder User, Verkehrsteilnehmer, Privateigentümer oder Eigentumsloser, was auch immer. Droht ein Konflikt zu eskalieren, ohne dass für Leib und Leben Gefahr besteht, signalisieren Menschen die Verhaltensgrenzen, die sie eingehalten haben wollen, indem sie spielerisch ein Lachen oder Weinen anzeigen, meistens in Gesten der Höflichkeit verwoben.

6 Siehe zu Plessners Theorie des Spielens und Schauspielens von Personenrollen insgesamt Krüger (1999, 4.-6. Kap.). 
Es gibt aber darüberhinausgehend kritische Situationen, in denen wir ungespielt ins Lachen oder Weinen geraten. Wir haben und gewinnen dann keine auf die Verhaltenssituation passende Antwort in dem Sinne, dass wir ihr angemessen handeln, sprechen und variabel gestalten können. Was wir uns hier und jetzt auch als Lösung für das Problem vorstellen, alle unsere Antwortmöglichkeiten auf die in Frage stehende Situation widersprechen sich und keine passt wirklich in die Situation. Wir sind paralysiert von sich widersprechenden Handlungsmöglichkeiten, die alle irgendwie an der Situation vorbeigehen. Diese Lagen bilden für Plessner Anlässe zum Lachen, insoweit sich die Betroffene aus der Lage, die sie soeben noch ernsthaft zu beantworten suchte, plötzlich herauszusetzen vermag. Die Palette des Lachens kann von einem kurzen bis zu einem langen ausfallen, womöglich sogar in einen langen Prozess ausarten, in dem die Betroffene am Ende einem Lachkrampf unterliegt. Es ist so, als ob uns der Leib im Lachen diejenige Antwort abnähme, die wir als Person im Augenblick nicht durch rollengemäßes Handeln, Sprechen und variables Gestalten zustande bringen.

Wir können aber auch in der entgegengesetzten Richtung die Selbstbeherrschung als Person verlieren. Dieses Mal nicht deshalb, weil wir eigentlich überschüssige Handlungsmöglichkeiten gegenüber der Situation haben, bloß dass diese sich widersprechen und im Ganzen nicht zur Situation passen, insofern wir auf Abstand zu ihr gehen. Es kann Grenzsituationen geben, indem überhaupt Sinnverlust eintritt, weil die Situation im Ganzen in keine Verhältnismässigkeit mehr passt und die betroffene Person nicht über den situativen Anlass hinausgelangen kann. Ihr ganzes Erwartungsraster für die Lebensführung rutscht ihr in die für sie außergewöhnliche Situation, aus der sie nicht mehr herausfindet. Es gibt dann hier und jetzt überhaupt keine dem Problem angemessene Antwort durch Handeln, Sprechen, Gestalten. Was soll man auch schon im Angesichte etwa des plötzlichen Todes eines geliebten Menschen tun, sagen, gestalten, um der Situation gerecht werden zu können? Man gerät nicht wie im Lachen außer sich in die Welt hinaus. Man fällt umgekehrt aus den Interaktionen in der Welt auf sich, seinen Leib zurück und bricht schließlich im Organismus zusammen.

Sowohl im ungespielten Lachen als auch im ungespielten Weinen bricht das Normalverhalten von Personen, die mehr oder minder beherrscht ihre Rollen spielen können, zusammen. In diesem Zusammenbruch sieht und hört man gut, was Menschen als personale Lebewesen üblicherweise für Verschränkungen zwischen Leibsein und Körperhaben in ihrem Verhalten in Anspruch nehmen. Sie benötigen eine Art und Weise von Integration zwischen Physis, Psyche und Geist im Handeln, Sprechen und Gestalten als Antwort auf Verhaltenssitua- 
tionen. Aber genau diese Antwort durch individuell variables Verschränken nach Rollenmustern bekommt die Person in Grenzsituationen nicht mehr zustande, sofern sie ungespielt ins Lachen oder Weinen fällt. Deshalb laufen ihr, was man umgangssprachlich auch so nennen kann, ihre Physis, Psyche und ihr Geist auseinander. Ihr selbstbestimmtes und selbstbeherrschtes Verhalten bricht zusammen. Sie kann nicht geistig angemessen reagieren, weil alles, was sie an kollektiver Intentionalität in ihrer Gesellschaft und Kultur gelernt hat, zu der außergewöhnlichen Situation nicht passt. Sie findet darauf auch als individuelle Psyche keine richtige Antwort, sei es, weil sie die kollektive Intentionalität zu sehr teilt, sei es, weil auch alle ihre Abweichungen davon keine Lösung hier und jetzt ermöglichen. Die einzige Möglichkeit zu antworten führt im ungespielten Lachen zu Prusten und Gurgeln, zu Luftnöten und Krämpfen, zur Ansteckung anderer, im ungespielten Weinen zu weichem Knie, Schwindel, $\mathrm{Zu}$ sammensacken, Abschottung und Vereinsamung, Schluchzen und Tränen. Die physischen Reaktionen stehen den Schweißgebadeten vor Augen und führen oft einen anonymen Mechanismus mit sich, nachdem die Person ins Lachen gefallen ist oder sich ins Weinen hat fallen lassen. Es ist merkwürdig, alle Menschen können lachen und weinen, diese Phänomene sind universell, und doch lacht jeder und jede anders als andere, weint jeder und jede anders als andere. Diese Phänomene sind in ihrer Universalität zugleich hoch individuell.

Damit haben wir eine gewisse Vorstellung von der leiblichen Grenze des soziokulturellen Rollenspiels der Personen gewonnen. Die Person verliert in der Abgabe der Antwort auf die Grenzsituation an den Leib, der lacht oder weint, ihre Selbstbeherrschung. Gleichwohl kann eine Person nicht anders leben als dadurch, dass sie in ihrem Leib ist und ihn als Körper hat. Im ungespielten Lachen und Weinen kann sie ihren Leib nicht mehr instrumentieren, indem sie ihn als Körper nach Rollenmaßstäben hat. Der Leib antwortet anstelle der Person, wodurch diese sich rettet. Aber auch er, der Leib, bricht auseinander. Statt die Einheit des Organismus mit der Umwelt im Verhalten herzustellen, fliegt er im Lachen dem Organismus und seiner Einheit mit der Umwelt davon in eine unbestimmte Welt hinaus. Die Leibesbewegung prustet im Lachen in exzentrischer Richtung aus sich heraus und davon, während sie im Weinen umgekehrt aus der Welt kommend auch aus der leiblichen Einheit mit der Umwelt schließlich im Organismus zusammensackt. Dadurch treten im Zusammenbruch - als der dialektischen Krisis - die beiden Richtungen des personalen Lebens hervor, einerseits im Lachen die Exzentrierung aus der Konzentrik des Leibes in die Welt hinaus, andererseits im Weinen die Rezentrierung der Welt auf den Leib, der schließlich in den Organismus hineinfällt. Normalerweise steht personales Leben in der Aufgabe, die exzentrierende und die rezentrierende Verhaltungss- 
richtung zu verschränken. Diese spannungsvolle Aufgabe nennt Plessner die exzentrische Positionalität, die einer „natürlichen Künstlichkeit“ (Plessner 1975, 309f.), „vermittelten Unmittelbarkeit“ (ebd., 321f.) und eines „utopischen Standortes“ (ebd., 341f.) bedarf.

Zusammenfassend schreibt Plessner in seiner Einführung ins Thema des Lachens und Weinens als Grenzerfahrungen:

Im Gegensatz zur mimischen Ausdrucksgebärde stellt sich das Genus von Lachen und Weinen als eine Äußerungsweise dar, bei welcher der Verlust der Beherrschung im Ganzen Ausdruckswert hat. Die Desorganisation des Verhältnisses zwischen dem Menschen und seiner physischen Existenz wird zwar nicht gewollt, aber - indem sie sich überwältigend einstellt - doch nicht bloß hingenommen und erlitten, sondern als Gebärde und sinnvolle Reaktion verstanden. In der Katastrophe noch, die sein sonst beherrschtes Verhältnis zum eigenen Leib erfährt, triumphiert der Mensch und bestätigt er sich als Mensch. Durch das entgleitende Hineingeraten und Verfallen in einen körperlichen Vorgang, der zwanghaft abläuft und für sich selbst undurchsichtig ist, durch die Zerstörung der inneren Balance wird das Verhältnis des Menschen zum Körper in eins preisgegeben und wiederhergestellt. Die effektive Unmöglichkeit, einen entsprechenden Ausdruck und eine passende Antwort zu finden, ist zugleich der einzig entsprechende Ausdruck, die einzig passende Antwort. (Plessner 1982f, 274)

Wenn wir nun abschließend die kulturphilosophische mit der naturphilosophischen Grenzauffassung vergleichen, dann fällt auf, dass die naturphilosophische Grenzbestimmung am lebenden Körper einsetzt. Insofern er seine eigene Grenze im Verhaltensvollzug realisiert, organisiert er sich nach innen und positioniert er sich nach außen. Die kulturphilosophische Grenzbestimmung setzt in dem Verhaltensspektrum ein, das zwischen der Exzentrierung und der Rezentrierung in der personalen Lebenssphäre liegt. Die Person hat normalerweise einen exzentrischen Abstand zu ihrer leiblich-konzentrischen Einheit von Organismus und Umwelt. Insofern sie die Distanz zum eigenen Leib hat, kann sie ihn nach Rollenmaßstäben instrumentieren und als Medium verwenden, d. h. als Körper haben. Aber im ungespielten Lachen und Weinen verliert sie diesen Abstand, sei es, dass sie in exzentrischer Richtung von der leiblichen Einheit mit der Umwelt gleichsam davonfliegt, sei es, dass sie in rezentrischer Richtung am Ende in ihren Organismus hineinsackt. Kam die naturphilosophische Grenze dem Organismus als belebtem Körper zu, im Unterschied zu unbelebten Körpern, liegt die kulturphilosophische Grenze im Verhaltensspektrum der exzentrischen Positionalität, das aber letztlich am Organismus des personalen Lebens hängt. Das soziokulturelle Verhalten gibt in seinem Zusammenbruch seine organismischen Randbedingungen doch wieder zu erkennen. Soziokulturelles Verhalten, das die Organismen nicht in leibliche Verhaltenseinheiten von Personen zu integrieren vermag, scheitert. Man kann darüber lachen oder weinen. 
Ungespieltes Lachen und Weinen können nicht verstetigt werden, ohne dass sie in Pathologien erstarren. Sie brauchen eine soziokulturelle Änderung der Rollen für Personen und der Individualisierungsmöglichkeiten in derartigen Rollen. Die Grenze der Kultur schlägt zurück in eine Grenze der Natur von Personen als Lebewesen. Unmenschlich wird es, wenn wir überhaupt nicht mehr lachen und weinen können, oder wenn wir ununterbrochen lachen und weinen müssen. Der Grenzübergang zwischen der Exzentrierung im Lachen und der Rezentrierung im Weinen muss dem personalen Leben als seine fortwährende Ermöglichungsstruktur angehören. Durch ihn hindurch lernt sich personales Leben selbst kennen, kommt es in seinen Grenzen zu sich. Was den Bruch zwischen Konzentrik und Exzentrik in der exzentrischen Positionalität auszeichnet, manifestiert sich in den sinnlich-sinnhaften Verhaltungsrichtungen des Lachens und des Weinens. Sie sind der personalen Lebenssphäre nicht zufällige, sondern wesentliche Grenzen ihres Verhaltensspektrums. - Sind uns dieses, nicht nur gespieltes, sondern auch ungespieltes Lachen und Weinen schon vergangen? 